\title{
A Novel Biologically Inspired Neural Network and Its Application on the Locomotion Control of Quadruped Robot
}

\author{
Lei Sun ${ }^{1, a}$, Yan $\mathrm{Cao}^{2, \mathrm{~b}}$ and Dequan Zhu ${ }^{1}$ \\ ${ }^{1}$ Department of technology, University of Anhui Agriculture, Hefei, Anhui, 230031, China \\ ${ }^{2}$ Dept. 6rd,New Star Research Inst. Of Applied Tech, Hefei, Anhui, 230031, China \\ ${ }^{\mathrm{a}} \mathrm{SL961102@163.com,}{ }^{\mathrm{b}}$ Knight.I.sun@gmail.com
}

Keywords: CPG; Proprioception; reflex; locomotion; quadruped robot.

\begin{abstract}
This paper proposed a novel Biologically Inspired Neural Networks-central pattern generator (CPG) model with proprioceptive and reflex mechanism. It not only contains inner sensory information but also contains the information of the actuators and automatically tunes the parameter of CPG correspond to the actuators information and inner sensory information. The position of the joints linked directly with the output of CPG is introduced to the CPG to found its proprioceptive system, spontaneously making the robot can realize the actuator working status, furthermore changing CPG output to fit the change and decrease influence of the problematic joints or actuators on the robot controlled. The reflex mechanism can tune the CPG output to fit the environment to avoiding obstacle and adapting the ground. Its application on the locomotion control of a quadruped robot demonstrates the effectiveness of the proposed approach.
\end{abstract}

\section{Introduction}

In this paper introduces a novel CPG with proprioception mechanism Reflex. It is proposed for the locomotion of quadruped that has a few sensors, which based on the basic concept of CPG [1][5]. Different with the previous CPGs, it introduces the joint motor information linked directly with the output of CPG and the Reflex into the oscillator networks. With this mechanism, it has the proprioception function by mastering the status of its actuators. When an actuator or some actuators have fault and don't work but others are fine, CPG must tune the oscillator unit controlling the wrong actuator to stop or change its output pattern. But the impact of other oscillator units on the whole CPG are still exit and greater than the problematic one if the connectivity mechanism of CPG network is static. In this case, the CPG can not tune its output better to cope with the situation. So the novel CPG adopts the dynamic connectivity mechanism, and decreases or removes the impact brought by other oscillators. When an oscillator with the proprioception mechanism feels that the actuator is wrong, it can produce lateral inhibitory, and make the oscillator unit linking with the abnormal actuator more excited and restrain the impact brought by other units. With the reflex signal, the legged robot can move on complex terrain (uneven ground, obstacles) and can tune the parameters to implement walking up and walking down hill using the pitch angle as the sensory feedback signal, dynamic tuning motion and obstacle avoiding using the laser radar signal as the sensory feedback signal.

We apply our system to control a simulated quadruped robot TIM-I. In particular, we show how the actuator information can be integrated to the CPG to make it has the proprioceptive mechanism, and introduce the reflex signal into the CPG to increase the CPG adaptive ability. The experiment Result demonstrates the effectiveness of the proposed approach.

\section{A CPG with proprioception and dynamic connectivity mechanism}

In a quadruped robot locomotion control system, when a joint has been damaged or it has other problems and the joint can't move, if it hasn't the proprioceptive mechanism, the CPG networks still output the locomotion wave signal, then the joint will be injured or re-injured. So CPG must tune its output by using the proprioceptive system. 
Nathaniel N Urban found the dynamic connectivity mechanism of cerebrum in 2008[2]. The neural networks can feel and discriminate the out stimuli when the cerebrum is under the "dynamic connect" status. When the cerebrum receives the out stimuli, many neurons output signal. But it is not a good phenomenon that many neurons output signal at the same time. In this process, the cerebrum can produce the lateral inhibition mechanism, and the stimulated neuron can translate the signal to the neighbored neurons and tell them to stop output signal. Urban and his group pointed that the connectivity of neurons aren't decided in advance but changed dynamically based on the out stimuli. The dynamic connectivity strengthens the lateral inhibition and filtrate other neurons' noise when lots of neurons react to the out stimuli.

We used the Hopf oscillator based on the oscillator proposed by Ijspeert [3]. A CPG is made of many oscillators linked together. We coupled the oscillators to generate the gaits needed by the quadruped robot locomotion control. The equation is as follow:

$$
\begin{aligned}
& \dot{v}_{i}=\alpha\left(\text { inhibi }_{i} \cdot \mu-\sqrt{x_{i}^{2}+v_{i}^{2}}\right) \square v_{i}-\omega x_{i} \\
& \dot{x}_{i}=\alpha\left(\text { inhibi }_{i} \cdot \mu-\sqrt{x_{i}^{2}+v_{i}^{2}}\right) x_{i}+\omega \cdot \text { motor }_{i}+\text { inhibi }_{i} \cdot \frac{\sum k_{i j}\left(x_{j}+v_{j}\right)}{x_{j}^{2}+v_{j}^{2}}+\text { feed }_{i} \\
& \text { inhibi }_{i}=F\left(\phi_{i}\right), \quad \phi_{i}=\mid \text { motor }_{i}-v_{i-1} \mid, \quad i=1,2, \cdots n \\
& v=v-v_{0}, v_{0}=\left\{\begin{array}{cc}
-\lambda \beta, & i=1,2 \\
\lambda \beta, & i=3,4
\end{array}\right.
\end{aligned}
$$

Here $n$ is the numbers of oscillators; $\omega, \alpha, \sqrt{\mu}$, motor are the same as forenamed meaning. $k_{i j}$ is the connection weight between the ith and jth oscillators. If $k_{i j}=0$ it means that oscillators $i$ and $j$ are not connected. ${ }^{v_{0}}$ is the mid-position of output. ${ }^{\text {inhibi }} i_{i}$ is the lateral inhibition variable that the $i$ th oscillator filtrates other oscillators' output on it; $F$ is the function of absolute value of the motor and $v$. It can be the S-function or others. Here it has the saturation characteristic. Equation (5) shows the F-function.

$$
F=\left\{\begin{array}{rr}
1 & 0<\phi \leq a, 0<a<b \\
\frac{b-\phi}{b-a}, & a<\phi<b \\
0 & b \leq \phi
\end{array} \quad(0 \leq a \leq b)\right.
$$

feed $_{i}$ is the sensory feedback of the exteroceptive senses. It can improve the movement ability of the quadruped robot in varies environment. With it the CPG can tune its output based on the environment information. So the CPG network can be strongly coupled with the robot it controls.

\section{CPG Control System of a quadruped robot}

CPG is a network which is comprised of many oscillators. A DOF is usually controlled by an oscillator. But the realtime performance will decrease if there are many oscillators in a CPG network. So this paper only considers the sagittal plane locomotion. Because there is the constant phase relation between the trajectory of the hip and the trajectory of the knee of the same leg, and to keep the locomotion as simple as possible, here the CPG only generates the joints angles of the hip of each leg. We choose the structure showed in Fig.1.

$\mathrm{LF}$ is the left front leg. $\mathrm{LH}$ is the left hind leg. RF is the right front leg. $\mathrm{RH}$ is the right hind leg. The connection matrix $k_{i j}$ decides the quadruped robot gait type. 


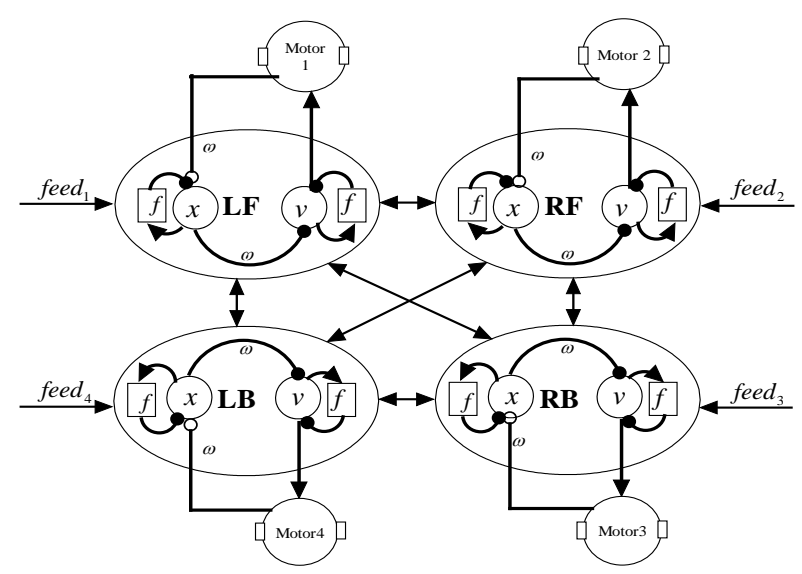

Fig. 1. Structure of the CPG for the quadruped robot.

\section{Experiments}

The system composed of the bio-inspired neural network and rigid dynamics modules has more complex dynamic properties and is difficult to be analyzed and controlled with normal control method. We constructed experiments based on Matlab software and Webots software. Webots is developed by Cyberbotics Ltd. It is 3D mobile robot simulation software and has a number of essential features to make the simulation easy to use and powerful. It is easy to model the legged robot with it, and it also includes a complete library of sensors and actuators. With the ODE (Open Dynamics Engine) library, users can simulate the kinetics and dynamics of the robot [4]. Matlab is famous with its numerical computing ability. So the CPG network's algorithm is implemented in Matlab. Matlab receives the enviorment and actuators' information from Webots via TCP/IP interface, and then computes the algorithm and translates the results to the Webots.

A. Locomotion in normal condition

First we consider the normal condition, and there is no accidental fault added to the robot in this condition. We adopt the CPG with the . proprioceptive and reflex mechanism to control the locomotion of the quadruped robot. Fig. 2 is the output simulation joints curves of matlab, and Fig.3 is the simulation video in Webots. From them we can conclude that the CPG with proprioception is suitable for the quadruped robot locomotion in normal condition.
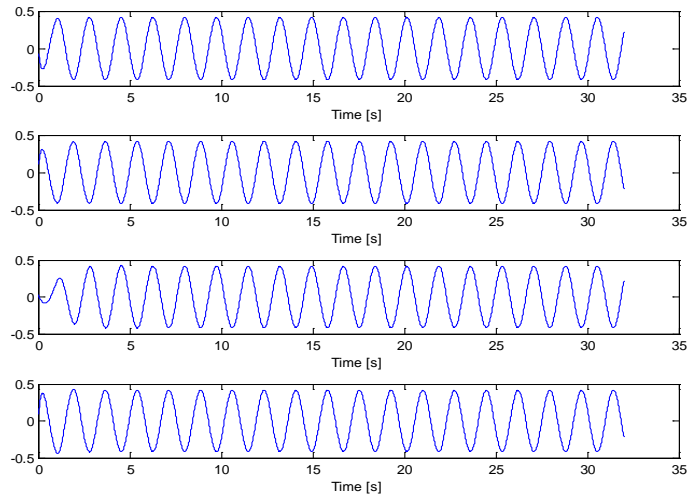

Fig.2. The curve of the trot pace

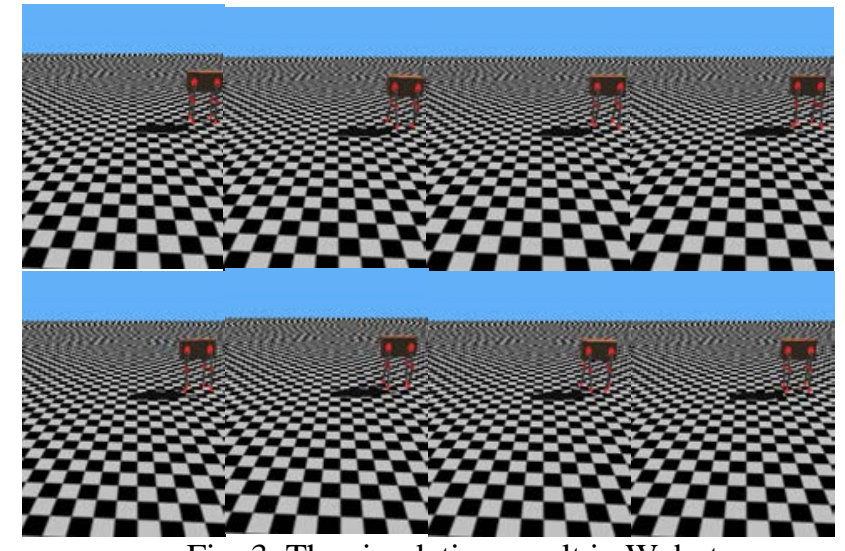

Fig. 3. The simulation result in Webots

B. Locomotion with accidental fault

Second, we consider the condition that the accidental fault added to the quadruped robot. The RF hip motor is blocked at the 150 step and the others are fine. Fig. 4 is the CPG output. When accidental fault added to the RF hip joint, the RF's oscillator tunes its output to stop quickly to response to the condition, and the others follow this change. Fig. 5 is the simulation experiment video in Webots. The quadruped robot stops moving and maintains its pose when accidental fault happened. 

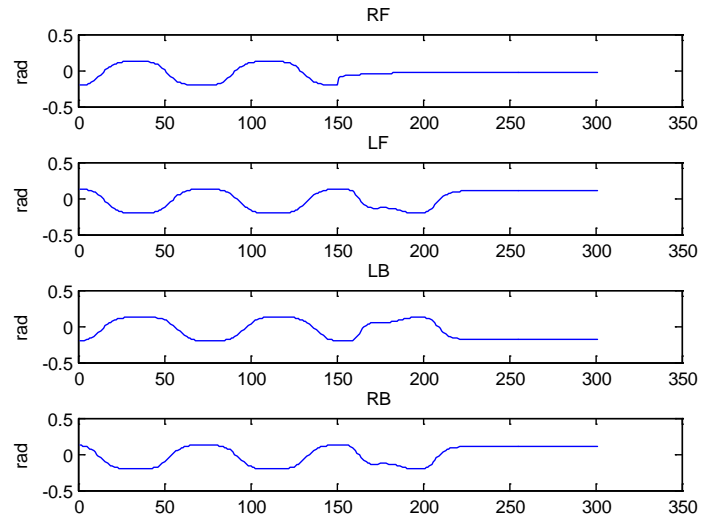

Fig. 4 The CPG output with accidental fault.

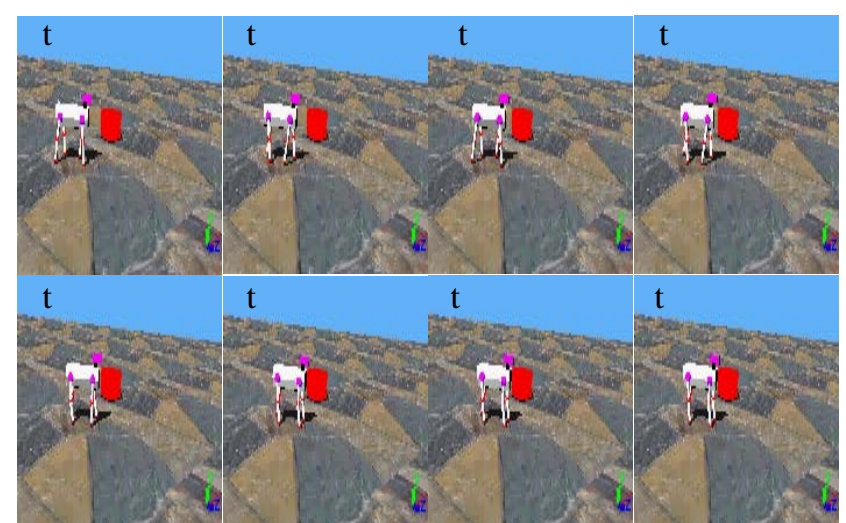

Fig. 5 The simulation experiment video with accidental fault

\section{Conclusions}

The main contribution is that we introduce the proprioception into the CPG, although the proprioception is simple and primary. Only the position of the joint is introduced. But this is important while the robot is moving. It can prevent the robot from overturning when some joint motors don't work or other accidental fault happened or can prevent the motor working at the block condition for a long time. With the proprioception, the CPG can get the information of the actuator it controlled and gives a right response quickly. However, there are many works to do on it. For instance, the function of the lateral inhibition variable $F$. Its choice is complex and has great influence on the performance of CPG. In the future works, we will test the method on the real physics quadruped robot TIM-I. And we will also study how to introduce the more complex proprioception into the CPG and how to integrate the reflex signal feedback with the proprioception.

\section{References}

[1] M. Porez, F. Boyer and A. J. Ijspeert. Improved Lighthill fish swimming model for bio-inspired robots: Modeling, computational aspects and experimental comparisons, in International Journal Of Robotics Research, vol. 33, num. 10,(2014), pp. 1322-1341

[2] Armen C Arevian, Vikrant Kapoor \& Nathaniel N Urban,“Activity-dependent gating of lateral inhibition in the mouse olfactory bulb”, Nat Neurosci. Jan; 7(1),( 2008) pp: 80-87

[3] G.C. Nandi, A.J. Ijspeert, P. Chakraborty, and A. Nandi.” Development of adaptive modular active leg (amal) using bipedal robotics technology". Robotics and Autonomous Systems, (2009),57(6-7):603-616

[4] O. Michel,“Webots: Professional mobile robot simulation,” International Journal of Advanced Robotic Systems, vol. 1, no. 1,(2007), pp: 39-42

[5] A.J. Ijspeert and A. Crespi, "Online trajectory generation in an amphibious snake robot using a lamprey-like central pattern generator model”. In Proceedings of the 2007 IEEE International Conference on Robotics and Automation,(2007), pp: 262-268 